\title{
THE TOTAL SQUARED CURVATURE OF CLOSED CURVES
}

\author{
JOEL LANGER \& DAVID A. SINGER
}

According to well-known arguments [1], a closed geodesic $\gamma$ in a positively curved sphere $M$ appears as a "minimax" critical point of the length functional $\mathfrak{L}$ and owes its existence to the higher homotopy of $M$. Alternatively, one could regard $\gamma$ as a curve which yields a minimum, hence a stable equilibrium, for the total squared geodesic curvature functional $\mathfrak{F}$. Indeed, a (nongeodesic) circle on a round sphere is carried to a point under the flow of " $-\nabla \mathfrak{Q}$ ", yet is carried to a nontrivial geodesic under the flow of “ $-\nabla \mathfrak{F}$ ". One motivation for the present investigation is to gain insight into the question of what should happen under the latter flow to an arbitrary closed curve on the sphere or on another manifold $M$.

Thus, we undertake here to describe the set of critical points of $\mathfrak{F}$ defined on the regular closed curves in some concrete manifolds $M$, to examine the stability of these critical points, and to seek relationships among the various critical points.

Of course, the study of the total squared curvature of curves is not new. A classical elastica, following Daniel Bernoulli's model of an elastic rod in equilibrium, is a curve in $R^{2}$ or $R^{3}$ which is critical for $\mathfrak{F}$ defined on regular curves of a fixed length satisfying given first order boundary data (for historical references concerning the classical elastica, we refer the reader to the recent survey by Truesdell [11]).

Here we introduce the term free elastica to describe the critical points which result when the constraint on arclength is removed; these are among the Euclidean curves studied by Radon (whose work is described in Blaschke's Vorlesungen über Differentialgeometrie. I). Much more recently, Bryant and Griffiths [2], [5] considered the natural generalizations of the elastica and the free elastica to space forms, and showed how the general theory of exterior differential systems leads naturally to the integration of the equations for these curves.

Received April 27, 1983 and, in revised form, April 13, 1984. 
The present paper also deals with the case $M$ a space form; however, the emphasis here is more on understanding in detail the nature of the solution curves themselves and drawing conclusions about the global behavior of $\mathfrak{F}$ - particularly behavior which ought to persist as the curvature of $M$ is perturbed from constant.

Consequently, the focus of attention here depends on the sign of the curvature of $M$. If $M^{2}$ has curvature $G \geqslant 0$, our main conclusion is that any nongeodesic closed free elastica is unstable (Theorem 3.1), and that these "wavelike" elasticae can be viewed as "minimax" solutions arising from certain pairs of distinct multiple covers of a prime geodesic. Thus, in answer to the earlier question, one expects almost any initial curve on the sphere to be carried to a geodesic under the $\mathfrak{F}$-decreasing flow.

In the case $G<0$, the greater complexity of the group of symmetries leads to a correspondingly richer qualitative description of free elasticae. Here, in addition to the closed geodesics and wavelike elasticae (which occur only when $M^{2}$ has nontrivial fundamental group), there arise free elasticae which lie entirely within annuli, and, as a limiting case, a circular free elastica $\varepsilon$ which achieves a global minimum for $\mathfrak{F}$ in the class of null-homotopic curves. Thus, we obtain the inequality $\int_{\gamma} k^{2} d s \geqslant 4 \pi \sqrt{-G}$, our main result (Theorem 4.1) for the case $G<0$.

There are several surprising facts relating to this result, one of which is that for $m \geqslant 3$, the $m$-fold cover of $\varepsilon$ fails to minimize $\mathfrak{F}$ among curves regularly homotopic to it (Theorem 4.2). It is also worth noting here that the above inequality can be used to prove a special case of the Willmore conjecture on the mean curvature of tori in $R^{3}$ (see [6]). Finally, we mention that the authors have learned of a recent proof by Ulrich Pinkall establishing the inequality in hyperbolic space of arbitrary dimension.

The authors wish to thank Beryl Langer for the drawings in this paper.

\section{Variation formulas}

All manifolds, maps, etc., will be assumed $C^{\infty}$. For a Riemannian manifold $M$, the metric will be denoted by $\langle$,$\rangle , and the Riemannian connection by \nabla$. For vectorfields $X, Y, Z$ on $M$, we write the structural equations $\nabla_{X} Y-\nabla_{Y} X$ $=[X, Y]$ and $\nabla_{X} \nabla_{Y} Z-\nabla_{Y} \nabla_{X} Z-\nabla_{[X, Y]} Z=R(X, Y) Z$, where $[$,$] is the$ Lie bracket, $R$ the Riemann curvature tensor.

We consider immersed curves $\gamma=\gamma(t): I \rightarrow M . V=V(t)$ will denote the tangent vector to $\gamma, T=T(t)$ the unit tangent, and $v$ the speed $v(t)=\|V(t)\|=$ $\langle V(t), V(t)\rangle^{1 / 2}$. The squared curvature $k^{2}(t)$ of $\gamma$ is given by $k^{2}=\left\|\nabla_{T} T\right\|^{2}$. 
The letter $\gamma$ will also denote a variation $\gamma=\gamma_{w}(t)=\gamma(w, t):(-\varepsilon, \varepsilon) \times I \rightarrow M$ with $\gamma(0, t)=\gamma(t)$. Associated with such a variation is the variation vector field $W=W(t)=(\partial \gamma / \partial w)(0, t)$ along the curve $\gamma(t)$. We will also write $V=V(w, t), W=W(w, t), T=T(w, t), v=v(w, t)$, etc., with the obvious meanings.

We let $s$ denote arclength, and write $\gamma(s), k^{2}(w, s), V(s)$, etc., for the corresponding reparametrizations. Here $s \in[0, L]$, where $L=\mathfrak{L}(\gamma)=$ the arclength of $\gamma$.

The following lemma collects some elementary facts which facilitate the derivations of the variational formulas (the first identity is standard and the others follow consecutively).

Lemma 1.1. Using the above notation, the following assertions are true:

(1) $[V, W]=0$.

(2) $\partial v / \partial w=\left\langle\nabla_{T} W, T\right\rangle v=-g v$, where $g=-\left\langle\nabla_{T} W, T\right\rangle$.

(3) $[W, T]=g T$.

(4) $[[W, T], T]=-T(g) T=-g_{s} T$.

(5) $\partial k^{2} / \partial w=2\left\langle\nabla_{T} \nabla_{T} W, \nabla_{T} T\right\rangle+4 g k^{2}+2\left\langle R(W, T) T, \nabla_{T} T\right\rangle$.

We now consider the one-parameter family of functionals

$$
\mathfrak{F}^{\lambda}(\gamma)=\int_{0}^{L} k^{2}+\lambda d s=\int_{0}^{1}\left(k^{2}+\lambda\right) v d t
$$

(We include the Lagrange multiplier $\lambda$ in our formulas partly because the case of constrained arclength will be useful for the proof of Theorem 4.1.) Using Lemma 1.1 one obtains the derivative

$$
\begin{aligned}
\frac{d}{d w} \mathfrak{F}^{\lambda}\left(\gamma_{w}\right)= & \int_{0}^{L}\left\langle W, 2\left(\nabla_{T}\right)^{3} T+\nabla_{T}\left(3 k^{2}-\lambda\right) T+2 R\left(\nabla_{T} T, T\right) T\right\rangle d s \\
& +\left[2\left\langle\nabla_{T} W, \nabla_{T} T\right\rangle+\left\langle W,-2\left(\nabla_{T}\right)^{2} T+\left(\lambda-3 k^{2}\right) T\right\rangle\right]_{0}^{L}
\end{aligned}
$$

If one considers $\mathfrak{F}^{\lambda}$ on a manifold consisting only of regular closed curves or curves which satisfy given first order boundary data, then the above boundary terms drop out. In this case it follows that a critical point $\gamma$ of $\mathfrak{F}^{\lambda}$ will satisfy

$$
0=E=2\left(\nabla_{T}\right)^{3} T+\nabla_{T}\left(3 k^{2}-\lambda\right) T+2 R\left(\nabla_{T} T, T\right) T .
$$

From now on, we call a unit-speed curve $\gamma$ an elastica if it satisfies equation (1.1) for some value of $\lambda$, and a free elastica if it satisfies (1.1) with $\lambda=0$.

Application of the Frenet equations for $\gamma$ to equation (1.1) leads to the equations and formulas we will find most useful. For instance, if $\gamma$ is a regular curve in an oriented 2-manifold $M, k$ is the signed curvature of $\gamma$, and $G(p)$ the Gaussian curvature of $M$ at $p$, then $\nabla_{T} T=k N$ reduces (1.1) to

$$
0=2 k_{s s}+k^{3}+2 k G-\lambda k \text {. }
$$


Or suppose $\gamma$ is a regular curve in an $n$-manifold $M$ of constant sectional curvature $G, \gamma$ has curvatures $\left\{k_{1}=k>0, k_{2}=\tau>0, k_{3}, k_{4}, \cdots, k_{n-1}\right\}$ and Frenet frame $\left\{N_{0}=T, N_{1}=N, N_{2}=B, N_{3}, N_{4}, \cdots, N_{n-1}\right\}$. Then (1.1) and the Frenet equations $\nabla_{T} N_{i}=-k_{i} N_{i-1}+k_{i+1} N_{i+1}, i=0,1, \cdots, n-1$ (defining $\left.k_{0} N_{0}=k_{n} N_{n}=0\right)$, yield

$$
\begin{aligned}
& 2 k_{s s}+k^{3}-2 k \tau^{2}-\lambda k+2 k G=0 \\
& k^{2} \tau=\text { constant, } \\
& k_{i}=0, \quad i \neq 1,2 .
\end{aligned}
$$

We note that (1.3) is also derived in [5] (by a different approach). A consequence of (1.3) is that we need only consider 2 and 3-dimensional $M$ in the constant curvature case.

Finally, we obtain a second variation formula which we will need for the case of an elastica $\gamma$ in a 2-manifold $M$ of constant curvature $G$. Here we consider a normal variation $\gamma_{w}$ with variation vector field $W=\phi N$, and we denote the derivative with respect to arclength by either a dot above or subscript $s$ :

$$
\begin{aligned}
\left.\frac{d^{2}}{d w^{2}} \mathfrak{F}\left(\gamma_{w}\right)\right|_{w=0} & =\left.\int_{\gamma}\left\langle W, \nabla_{W} E\right\rangle d s\right|_{w=0} \\
& =\int_{\gamma} 2(\ddot{\phi})^{2}-\left(5 k^{2}+4 G-\lambda\right)(\dot{\phi})^{2}+A \phi^{2} d s \\
& =\int_{\gamma} \phi\left[2 \ddot{\phi}+\left(5 k^{2}+4 G-\lambda\right) \ddot{\phi}+10 k \dot{k} \dot{\phi}+A \phi\right] d s,
\end{aligned}
$$

where $A=6 \dot{k}^{2}-k^{4}-3(G-\lambda) k^{2}+G(2 G-\lambda)$.

Proof of (1.4). With $W=\phi N$, Lemma 1.1 gives $\nabla_{T} W=\dot{\phi} N-\phi k T, g=\phi k$, and $[W, T]=\phi k T$. Differentiating, one obtains $W(k)=\ddot{\phi}+\phi k^{2}+\phi G$ and $W(k)_{s s}=\ddot{\phi}+\ddot{\phi} k^{2}+4 \dot{\phi} k \dot{k}+2 \phi \dot{k}^{2}+2 \phi k \ddot{k}+\ddot{\phi} G$. Thus one easily computes

$$
\begin{aligned}
W(\ddot{k}) & =W T T k=T T W k+T[W, T] k+[W, T] T k \\
& =\dddot{\phi}+\ddot{\phi} k^{2}+5 \dot{\phi} k \dot{k}+3 \phi \dot{k}^{2}+4 \phi k \ddot{k}+\ddot{\phi} G .
\end{aligned}
$$

Using the substitution $\ddot{k}=-k^{3} / 2-G k+\lambda k / 2$ and the above formulas for $W(k)$ and $W(\ddot{k})$, one obtains

$$
\begin{aligned}
W(E \cdot N)= & 2 W(\ddot{k})+\left(3 k^{2}+2 G-\lambda\right) W(k) \\
= & 2 \dddot{\phi}+\ddot{\phi}\left(5 k^{2}+4 G-\lambda\right)+10 \dot{\phi} k \dot{k} \\
& +\phi\left(6 \dot{k}^{2}-k^{4}-3 G k^{2}+3 \lambda k^{2}+2 G^{2}-\lambda G\right) .
\end{aligned}
$$

Integrating by parts, the other formula follows. 


\section{Integration of the equations}

Let $M$ be a manifold having constant sectional curvature $G$. In this section we discuss the integration of the equations (1.3) as well as the Frenet equations for an elastica $\gamma$ in $M$. As noted above, there is no essential loss of generality in assuming $M$ has dimension 2 or 3 (in the 2-dimensional case we will want to consider the signed curvature, but we will not run into trouble by deriving all our formulas from (1.3) and simply regarding (1.2) as a special case).

We begin by combining the first two equations of (1.3): $0=k_{s s}+\frac{1}{2} k^{3}+$ $(G-\lambda / 2) k-c^{2} / k^{3}$, where $c$ is the constant $c=k^{2} \tau$. Multiplication by $2 k_{s}$ and integration yields

$$
\left(k_{s}\right)^{2}+\frac{1}{4} k^{4}+(G-\lambda / 2) k^{2}+c^{2} / k^{2}=A=\text { undetermined constant. }
$$

Making the change of variable $u=k^{2}$, we arrive at

$$
\left(u_{s}\right)^{2}+u^{3}+4(G-\lambda / 2) u^{2}-4 A u+4 c^{2}=0 .
$$

Since this equation is of the form $\left(u_{s}\right)^{2}=P(u), P$ a third degree polynomial, it can be solved by standard techniques in terms of elliptic functions.

In order to write down and analyze the solution to this equation, we first fix notation regarding elliptic functions. We begin with the elliptic integral of the first kind

$$
x(\phi)=\int_{0}^{\phi} \frac{d \theta}{\sqrt{1-p^{2} \sin ^{2} \theta}}, \quad 0 \leqslant p \leqslant 1 .
$$

Denoting the inverse of $x(\phi)$ by $\operatorname{am}(x, p)=\phi$, the Jacobi elliptic functions are given by

$$
\operatorname{sn} x=\operatorname{sn}(x, p)=\sin \phi, \operatorname{cn} x=\operatorname{cn}(x, p)=\cos \phi,
$$

and

$$
\operatorname{dn} x=\operatorname{dn}(x, p)=\sqrt{1-p^{2} \sin ^{2} \phi} .
$$

We have periodicity relations $\operatorname{sn}(x+2 K)=-\operatorname{sn} x, \operatorname{cn}(x+2 K)=-\operatorname{cn} x$, $\operatorname{dn}(x+2 K)=\operatorname{dn} x$, where $K=x(\pi / 2)$ - the complete elliptic integral of the first kind. To list a few of the other basic identities,

$$
\begin{aligned}
& \operatorname{sn}^{2}(x, p)+\operatorname{cn}^{2}(x, p)=1=p^{2} \operatorname{sn}^{2}(x, p)+\operatorname{dn}^{2}(x, p), \\
& \frac{d}{d x} \operatorname{sn} x=\operatorname{cn} x \operatorname{dn} x, \quad \frac{d}{d x} \operatorname{cn} x=-\operatorname{sn} x \operatorname{dn} x, \\
& \frac{d^{2}}{d x^{2}} \operatorname{sn} x=2 p^{2} \operatorname{sn}^{3} x-\left(1+p^{2}\right) \operatorname{sn} x
\end{aligned}
$$

etc. 
Let us return now to equation (2.2). The cubic polynomial $P(u)$ satisfies $P(0)=-4 c^{2} \leqslant 0$, and $\lim _{u \rightarrow \pm \infty} P(u)=\mp \infty$. Furthermore, if $u=k^{2}$ is a nonconstant solution to (2.2), it must obviously take on values at which $P(u)>0$. It follows that we may assume $P(u)$ has three real roots $-\alpha_{1}, \alpha_{2}, \alpha_{3}$ satisfying $-\alpha_{1} \leqslant 0 \leqslant \alpha_{2} \leqslant \alpha_{3}$ (and treat the case $k=$ constant separately).

We can now write equation (2.2) in the form

$$
\left(u_{s}\right)^{2}+\left(u+\alpha_{1}\right)\left(u-\alpha_{2}\right)\left(u-\alpha_{3}\right)=0,
$$

and its solution is given by

$$
u=u(s)=\alpha_{3}\left(1-q^{2} \operatorname{sn}^{2}(r s, p)\right),
$$

where

$$
p^{2}=\frac{\alpha_{3}-\alpha_{2}}{\alpha_{3}+\alpha_{1}}, \quad q^{2}=\frac{\alpha_{3}-\alpha_{2}}{\alpha_{3}}, \quad r=\sqrt{\frac{\alpha_{3} q^{2}}{4 p^{2}}}=\frac{1}{2} \sqrt{\alpha_{3}+\alpha_{1}}
$$

(for background on the solution of such equations, see [4]). Of course, $\alpha_{1}, \alpha_{2}$, $\alpha_{3}$ are related to the coefficients of $P(u)$ by

$$
\begin{aligned}
& 4 G-2 \lambda=\alpha_{1}-\alpha_{2}-\alpha_{3}, \\
& 4 c^{2}=\alpha_{1} \alpha_{2} \alpha_{3} \\
& 4 A=\alpha_{1} \alpha_{3}+\alpha_{1} \alpha_{2}-\alpha_{2} \alpha_{3}
\end{aligned}
$$

Thus, it is clear that (treating $\lambda$ as a known fixed constant) $\alpha_{1}, \alpha_{2}$ can be determined if the maximum squared curvature $\alpha=\alpha_{3}=u(0)$ and minimum torsion $\beta=\tau(0)=\frac{1}{2} \sqrt{\alpha_{1} \alpha_{2} / \alpha}$ of the elastica $\gamma$ are given. The set of solutions $(u(s), \tau(s))$ can therefore be considered as a two-parameter space (one parameter in case $\operatorname{dim} M=2$ ). However, the parameters $\alpha, \beta \geqslant 0$ cannot be chosen arbitrarily; for they must yield real numbers satisfying $-\alpha_{1} \leqslant 0 \leqslant \alpha_{2} \leqslant \alpha_{3}$.

The situation is summarized by the following two tables, which are derived from (1.3), (2.4), (2.5), (2.6) and the above inequality. For simplicity of interpretation, only the free elastic curves are represented in the tables (for $\lambda \neq 0$, one needs only replace $2 G$ with $2 G-\lambda$ to recover the general case).

From Table (2.7) one sees, for example, that if $\gamma$ is a nongeodesic free elastica in the plane or the standard two-sphere, then $\gamma$ must be wavelike ( $k$ $=\sqrt{\alpha} \mathrm{cn}(r s, p)$, which behaves qualitatively like ordinary cosine). On the other 
TABLE (2.7): Free elastica $\gamma$ for $\tau \equiv 0$.

(a) $\quad G>0$

\begin{tabular}{|l|l|l|}
\hline$\alpha=0$ & $\gamma$ is a geodesic & \\
\hline$\alpha>0$ & $k^{2}=u=\alpha \operatorname{cn}^{2}(r s, p)$ & $0<p<\sqrt{1 / 2}$ \\
\hline
\end{tabular}

(b) $\quad G=0$

\begin{tabular}{|l|l|l|}
\hline$\alpha=0$ & $\gamma$ is a geodesic & \\
\hline$\alpha>0$ & $k^{2}=u=\alpha \operatorname{cn}^{2}(r s, p)$ & $p=\sqrt{1 / 2}$ \\
\hline
\end{tabular}

(c)

\begin{tabular}{|l|l|l|}
\hline$\alpha=0$ & $\gamma$ is a geodesic & \\
\hline $0<\alpha<-2 G$ & $\gamma$ does not exist & \\
\hline$\alpha=0$ & $k^{2}=$ constant $=-2 G$ & $p=q=0$ \\
\hline$-2 G<\alpha<-4 G$ & $k^{2}=u=\alpha \operatorname{dn}^{2}(r s, p)$ & $0<p=q<1$ \\
\hline$\alpha=-4 G$ & $k^{2}=u=-4 G \operatorname{sech}^{2}(r s)$ & $p=q=1$ \\
\hline$\alpha>-4 G$ & $k^{2}=u=\alpha \operatorname{cn}^{2}(r s, p)$ & $\sqrt{1 / 2}<p<1$ \\
\hline
\end{tabular}

TABLE (2.8): Free elastica $\gamma$ for $\tau>0$.

\begin{tabular}{|l|l|}
\hline$\alpha-2 \beta^{2}<-2 G$ & $\gamma$ does not exist \\
\hline$\alpha-2 \beta^{2}=-2 G$ & $k^{2}=$ constant $=-2 G+2 \beta^{2}$ \\
\hline$\alpha-2 \beta^{2}>-2 G$ & $k^{2}=u=\alpha\left(1-q^{2} \operatorname{sn}^{2}(r s, p)\right) \quad 0<p<q<1$ \\
\hline
\end{tabular}

hand, in the hyperbolic plane, a free elastica may also be orbitlike ( $k$ $=\sqrt{\alpha} \operatorname{dn}(r s, p)>0)$, circular $(k \equiv \sqrt{-2 G})$, or asymptotically geodesic $(k=$ $2 \sqrt{-G} \operatorname{sech}(r s))$. Note also that with $G<0$ there is a gap separating geodesics from the main continuum of elasticae. Finally, we observe that when $k$ is not zero (the case covered by Table (2.8)) neither $k$ nor $\tau$ ever vanish.

The appropriateness of the terms wavelike and orbitlike is only hinted at by the curvature functions, but becomes more apparent as further integration reveals the shapes of the elastic curves themselves, the subject we now take up. Our approach to this integration will be somewhat ad hoc, but will take us quickly to our main goals in this section-to describe the global geometry of elastic curves and to prepare for derivations of some formulas (in the appendix) needed for the following sections. A more systematic approach to the integration, making use of the theory of exterior differential systems, is described in [2] and [5] (although these references do not include most of the specific information we require). 
As regards the problem of integrating the Frenet equations for an elastica, the gist of our solution is contained already in

Proposition 2.1. Let $M$ be a simply connected manifold having constant sectional curvature $G$, and let $\gamma$ be an elastica in $M$. Then the vectorfields $J_{\gamma}=\left(k^{2}-\lambda\right) T+2 k_{s} N+2 k \tau B$ and $H_{\gamma}=k B$ extend to Killing fields $\tilde{J}_{\gamma}$ and $\tilde{H}_{\gamma}$ on $M$.

Proof. If $\gamma$ is a curve in a manifold $M$ let us call a vectorfield $W$ along $\gamma$ Killing along $\gamma$ if it annihilates $v$ and all $k_{i}$; to be specific, in the 3-dimensional case, the condition is that for any variation $\gamma_{w}$ in the $W$ "direction" (i.e., $\partial \gamma_{w} / \partial w=W$ ) one has $0=\partial v / \partial w=\partial k / \partial w=\partial \tau / \partial w$. Using parts (2) and (5) of Lemma 1.1 and the formula

$$
\frac{\partial}{\partial w} \tau^{2}=2\left\langle\frac{1}{k}\left(\nabla_{T}\right)^{3} W-\frac{k_{s}}{k^{2}}\left(\nabla_{T}\right)^{2} W+\left(\frac{G}{k}+k\right) \nabla_{T} W-\frac{k_{s}}{k^{2}} G W, \tau B\right\rangle
$$

one sees that the above condition constitutes a linear system in $W$ whose solution space is 6-dimensional in case $\operatorname{dim} M=3$, and 3-dimensional in case $\operatorname{dim} M=2$.

Now when $M$ is a simply connected manifold of constant curvature, these dimensions agree with the dimensions of the respective isometry groups. Thus, a Killing field along a curve $\gamma$ in $M$ is evidently the restriction to $\gamma$ of a Killing field on $M$. To finish the proof one need only check that if $\gamma$ happens to be an elastica and $W=J_{\gamma}$ or $W=H_{\gamma}$, the formulas for $\partial v / \partial w, \partial k / \partial w$, and $\partial \tau / \partial w$ yield 0. q.e.d.

Now suppose, for example, $\gamma$ is a nongeodesic free elastica on the 2-sphere. Since we are in the two-dimensional case $\gamma$ induces only the one Killing field $\tilde{J}_{\gamma}$ and we can think of the unique integral geodesic of $\tilde{J}_{\gamma}-$ i.e., the set of points at which $\left|\tilde{J}_{\gamma}\right|$ has a maximum-as the equator of $S^{2}$. Obviously, $J_{\gamma}=k^{2} T+2 k_{s} N$ is perpendicular to $\gamma$ (and nonvanishing) at inflection points of $\gamma$ (points at which $k$ changes sign) and is tangent to $\gamma$ at vertices of $\gamma$ (points at which $k$ has an extremum). Moreover, one easily computes $d\left|J_{\gamma}(s)\right|^{2} / d s=-8 G k k_{s}$, hence $\gamma$ crosses the equator precisely at its inflection points and looks qualitatively as shown in Figure 1(a).

To obtain quantitative information, observe that if $x$ and $y$ are coordinates of longitude and latitude on $S^{2}$, then the coordinate field $\partial / \partial x$ is simply $A \tilde{J}_{\gamma}$, where the normalization factor $A$ is chosen so that $A J_{\gamma}$ has unit length on the equator (in fact $A=1 / \sqrt{\alpha_{1} \alpha}$ ). So to obtain $x(s)$ one needs only integrate the known function $d x / d s=(T \cdot \partial / \partial x) /|\partial / \partial x|^{2}$; and since $|\partial / \partial x|=\cos y$, one can also obtain $y(s)$ in terms of elliptic functions. The elliptic integral $x(s)$ is analyzed in the appendix for the purpose of determining the possible "wavelengths" of $\gamma$, hence determining all closed $\gamma$. 


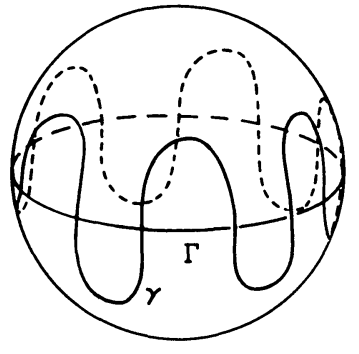

a)

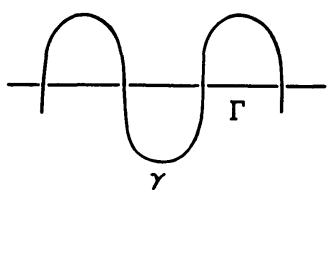

b)

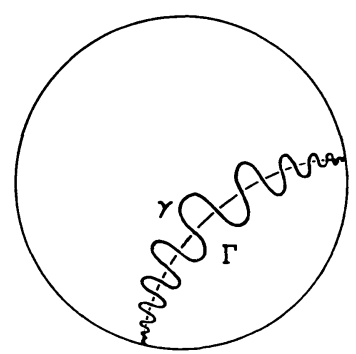

c)

FIG. 1

Wavelike free elastica $\gamma$ and axial geodesic $\Gamma$ on (a) the sphere, (b) the plane, (c) the Poincare disc

As suggested by Figure 1, a wavelike free elastica for $G \leqslant 0$ has a very similar description. Specifically, it oscillates along an axial geodesic, $\Gamma_{\gamma}$, which it crosses perpendicularly at each inflection point, and with respect to which it has the obvious symmetries.

Determining the shape of an elastica in the hyperbolic plane is just slightly more complicated than in the spherical case, owing to the greater variety of Killing fields and the fact that computing the normalization constant $A$ is not quite as trivial. The following two propositions suffice for dealing with these points (and will be needed also for the proof of Theorem 4.1).

Proposition 2.2. Let $\gamma$ be an elastica in $M^{2}$, and let $p=\gamma\left(t_{0}\right)$ be a vertex of $\gamma$. Then $J_{\gamma}$ is tangent to $\gamma$ at $p$, and the integral curve $\Sigma$ in $M$ of $\tilde{J}_{\gamma}$ through $\tilde{p}$ has curvature $k_{\Sigma}=-2 G k\left(t_{0}\right) /\left(k^{2}\left(t_{0}\right)-\lambda\right)$.

(We omit the proof, which is an easy computation.)

Proposition 2.3. Let $\gamma, \sigma, \varepsilon, \eta$ be wavelike, orbitlike, circular, and asymptotically geodesic (respectively) free elasticae in $M^{2}$. Then $\tilde{J}_{\gamma}$ is translational (has an integral geodesic), $\tilde{J}_{\sigma}$ is rotational (has a zero) and $\tilde{J}_{\eta}^{\gamma}$ is horocyclical (has an integral horocycle).

Proof. Apply Proposition 2.2 and Table (2.7), using the fact that in the hyperbolic case, $\tilde{J}$ is translational, horocyclical, or rotational according to whether $\Sigma$ is an equidistant curve, a horocycle, or a circle, in turn depending on whether $\left|k_{\Sigma}\right|^{2}$ is less than, equal to, or greater than $-G$. q.e.d.

With the help of the above proposition one easily sees, e.g., that an orbitlike free elastica $\sigma$ behaves qualitatively as shown in Figure 2(b), and one obtains the elliptic integral for the angular progress $\Delta \theta$ which $\sigma$ makes in each period (see the appendix), hence determining all closed orbitlike elasticae. 


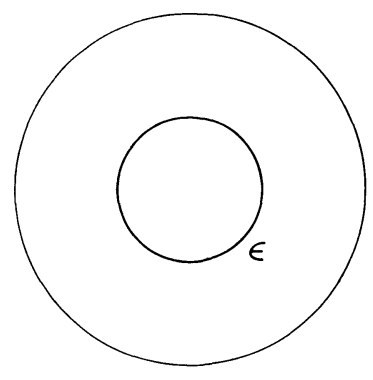

a)

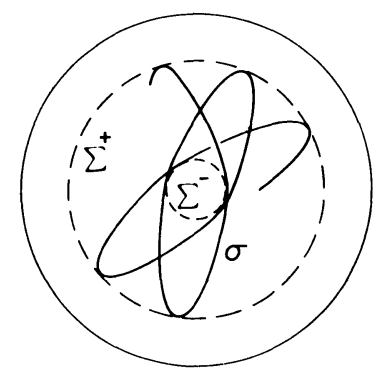

b)

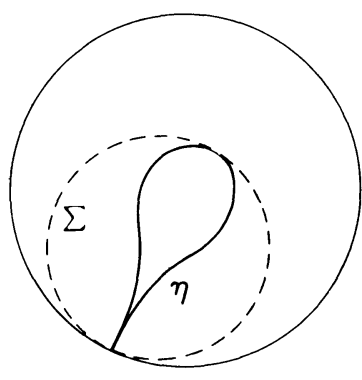

c)

FIG. 2

Poincare disc model of hyperbolic plane containing (a) circular free elastica $\varepsilon$, (b) orbitlike free elastica $\sigma$ oscillating between the pair of integral circles $\Sigma^{+}, \Sigma^{-}$of $\tilde{J}_{\sigma}$, (c) asymptotically geodesic free elastica $\eta$ with integral horocycle $\Sigma$ of $\tilde{J}_{\eta}$.

Above, we have applied Proposition 2.1 to understand the behavior of elastic curves in simply connected 2-manifolds of constant curvature. Of course, if $M$ is not simply connected one need only carry out the analysis on a lift of $\gamma$ to the universal cover of $M$ and then project back down. If $M$ is three-dimensional the analysis is somewhat more complicated, as one must work with two Killing fields, $\tilde{J}_{\gamma}, \tilde{H}_{\gamma}$, and compare them with a wider variety of Killing fields on $M$. However, it is essentially clear how to proceed in order to obtain the corresponding information in the three-dimensional case. Such an analysis is carried out in [7] for the case $M=R^{3}$, yielding a classification of all closed (nonfree) elastic curves in Euclidean space and a determination of their knot types.

\section{Minimax elasticae in the 2-sphere}

For determining the closed free elasticae in the 2-sphere all that was missing in the discussion of the previous section was an analysis of the possible values of the wavelength $\Lambda$ - the amount of progress the elastica makes along its axial geodesic (the equator in this case) in one period of $k$, as measured by arclength along the geodesic. In the appendix it is shown that in the case of the sphere $\Lambda$ ranges between 0 and $2 \pi$, and that for each $\Lambda, 0<\Lambda<2 \pi$, a wavelike free elastica (not necessarily closed) is uniquely determined (modulo isometries of $S^{2}$ ). Two cases near the upper and lower extremes are illustrated in Figure 3.

Of course, $\gamma$ closes up precisely when $\Lambda$ is rationally related to $\pi$. We conclude that the set of closed free elasticae on $S^{2}$ (excluding geodesics) is 


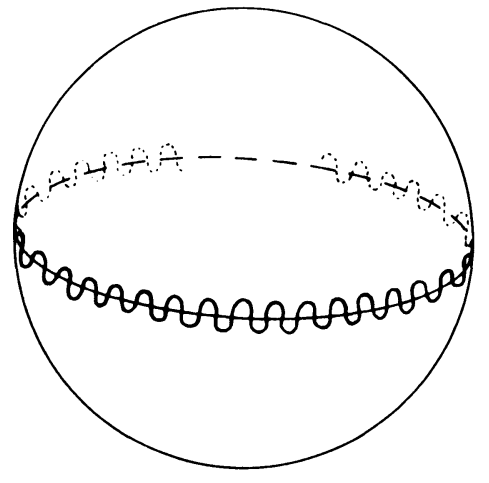

a)

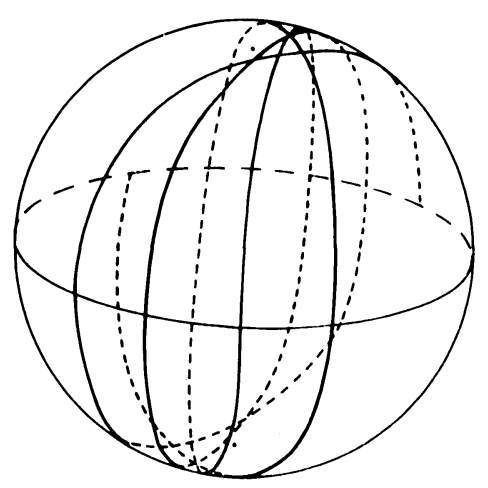

b)

FIG. 3

Wavelike free elastica on the sphere for (a) small wavelength $\Lambda$, and (b) wavelength near upper limit $\Lambda_{\max }=2 \pi$.

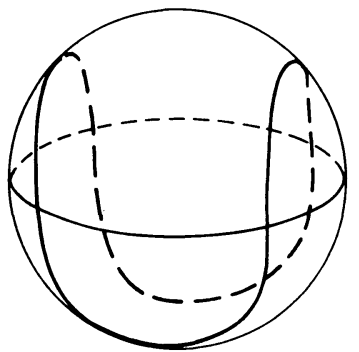

a)

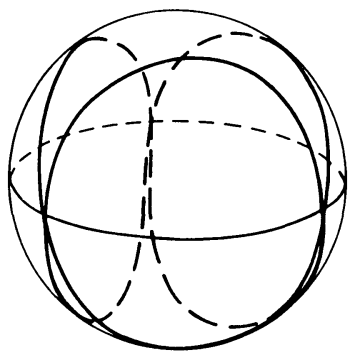

b)

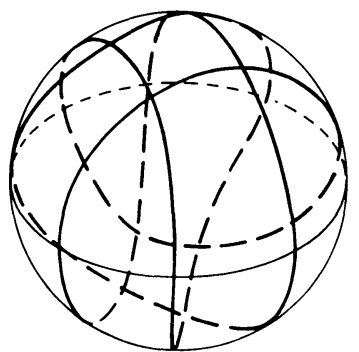

c)

Fig. 4

Closed free elastica on the sphere: $(a) \gamma_{1,2},(b) \gamma_{2,3},(c) \gamma_{3,4}$,

indexed one-to-one by pairs of integers, $0<m<n$, where $\gamma_{m, n}$ closes up after $n$ periods and $m$ trips around the equator. Three of the simplest $\gamma_{m, n}$ are shown in Figure 4.

There is a satisfying heuristic for the existence of the $\gamma_{m, n}$ as critical points of $\mathfrak{F}=\mathfrak{F}^{0}$ (defined on a space of immersed circles in $S^{2}$ ). Denote by $\mathbb{S}$ the group of symmetries of $\gamma_{m, n}$. (Abstractly, $\mathbb{S}$ has presentation $\mathfrak{S}=\left\{a, b: a^{2 n}=\right.$ $\left.b^{2}=b a b a=\mathrm{id}\right\}$.) Let $\Gamma^{m}$ and $\Gamma^{(2 n-m)}$ be, respectively, the $m$-fold and the $(2 n-m)$-fold coverings of the equator $\Gamma$. Then $\Gamma^{m}$ and $\Gamma^{(2 n-m)}$ are (5)equivariantly regularly homotopic. Figure 5 shows the homotopy of one $n$th of the curve. 


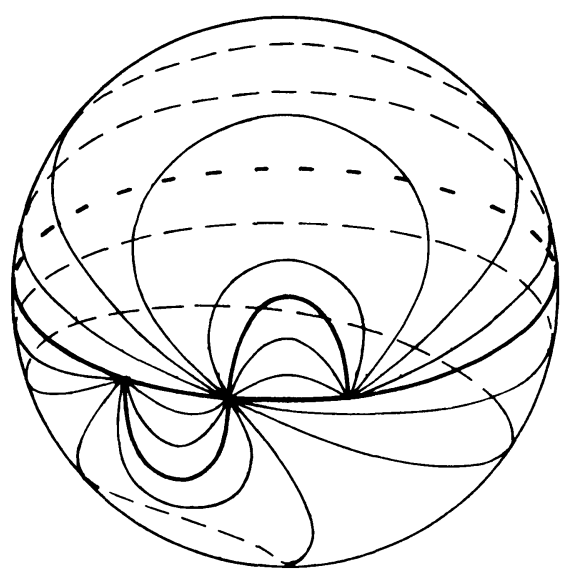

Fig. 5

Wavelike free elastica on the Poincare disc for (a) largest possible wavelength $\Lambda=b$, (b), (c) small wavelength - two solutions for the same value of $\Lambda$.

Now $\Gamma^{m}$ and $\Gamma^{(2 n-m)}$ are both minima for $\mathfrak{F}$, so one would expect a minimax argument, together with the "principle of symmetric criticality", to yield a third critical point having exactly the symmetry of $\gamma_{m, n}$. In fact, by the above description, it is clear that $\gamma_{m, n}$ is the only elastica which could arise in this way. Thus, the wavelike elasticae are precisely the critical points one would expect, other than the geodesics.

The above minimax argument is a strong hint in the direction of our main instability result:

Theorem 3.1. Let $M^{2}$ have constant curvature $G \geqslant 0$ and let $\gamma$ be a closed free elastica in $M$. Then $\gamma$ is stable if and only if $\gamma$ is a geodesic.

Proof. The main idea of the proof is to take as our variation vectorfield the " $L^{2}$-arclength gradient" $W=k N$. This essentially isolates the simpler of the two possible sources of instability of an elastica, the other one being that $\gamma$ may have "too many" inflection points and thus be unstable even for the constrained arclength problem. As will be apparent from the proof, if $\gamma$ is wavelike, the length gradient renders even a single arch of $\gamma$ unstable, provided $\gamma$ is allowed to pivot at the inflection points (see Figure 5).

With $\phi=k$ and the second of the two formulas in (1.4), straightforward computation gives

$$
\left\langle\nabla_{W} E, W\right\rangle=-9 / 2 k^{6}-16 G k^{4}+10 k^{2}\left(\alpha^{2} / 4+G \alpha\right) .
$$


For wavelike $\gamma$ we have $k^{2}=\alpha \mathrm{cn}^{2}(r s, p)$. Using this, and also using

$$
\begin{gathered}
r=\frac{\sqrt{\alpha}}{2 p}, \quad \alpha=\frac{4 G p^{2}}{1-2 p^{2}}, \quad C_{2}=\int_{0}^{K} \mathrm{cn}^{2} u d u, \quad C_{4}=\int_{0}^{K} \mathrm{cn}^{4} u d u, \\
C_{6}=\int_{0}^{K} \mathrm{cn}^{6} u d u=\frac{4}{5 p^{2}}\left(2 p^{2}-1\right) C_{4}+\frac{3}{5 p^{2}}\left(1-p^{2}\right) C_{2},
\end{gathered}
$$

one obtains a negative quantity for the second variation in the direction $W=k N$ over one arch (i.e., one half-period) of $\gamma$ :

$$
\delta^{2}=-\frac{4}{5} p \alpha^{3 / 2}\left[(\alpha+4 G) C_{2}+8 G C_{4}\right] \text {. q.e.d. }
$$

One may ask if the minimax argument itself might be made into a rigorous (and more satisfying) proof of Theorem 3.1. The main obstacle is to establish some version of the Palais-Smale Condition $\mathrm{C}$, which justifies such an argument in the infinite-dimensional manifold setting (see [9]). For the case of curves in $R^{3}$ under the constraint of fixed length, Condition $\mathrm{C}$ has been established and a very similar"symmetrical minimax" argument implied instability for all nonplanar closed elastic curves in Euclidean space [8].

In the present situation, however, it appears to be an interesting challenge to rule out the possibility that arclength tends to infinity on a trajectory of " $-\nabla \mathfrak{F}$ "; in fact, this can obviously happen in the case $G \leqslant 0$ ( $M$ compact or not). Nevertheless, we expect that on any manifold $M$ of positive sectional curvature (not necessarily constant), trajectories of " $-\nabla \mathfrak{F}$ " converge, and that the limits are almost always closed geodesics.

\section{The hyperbolic case}

We begin this section with a brief description of the closed free elastic curves in an orientable 2-manifold $M$ of constant negative curvature $G$. This will lead us to the main topic of this section, the inequality stated in the introduction, which gives the circular elastica a special status among closed curves in $M$.

Suppose first $\gamma$ is a wavelike free elastica. Since $\gamma$ oscillates along its axial geodesic $\Gamma$, it is clear that $\gamma$ cannot be closed unless $\Gamma$ is closed. In particular, $\gamma$ can never be closed if $M$ is the hyperbolic plane $H^{2}$, and more generally, it follows that if $\gamma$ is closed in $M$, then $\gamma$ cannot be null-homotopic.

Of course, the condition for closedness of $\gamma$ is that the wavelength $\Lambda$ is rationally related to the length of $\Gamma$. The behavior of $\Lambda$ as a function of the parameter $\alpha$ (maximum squared curvature of $\gamma$ ) is analyzed in the appendix, and in this case leads to a bit of a surprise: as $\alpha$ increases from its lower limit of $-4 G$ to $\infty$, the wavelength $\Lambda$ increases from 0 to some maximum value $b$ (approximately $2 / \sqrt{-G}$ ) and then decreases back down to 0 . Figure 6 illustrates this behavior in the Poincare disc. 


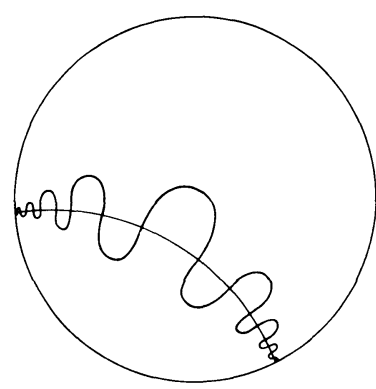

a)

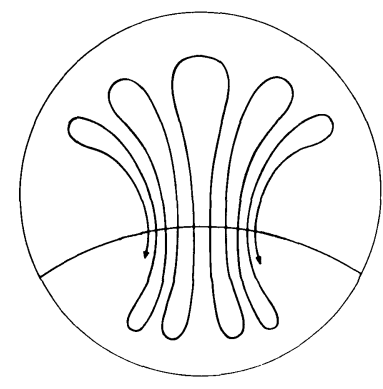

b)

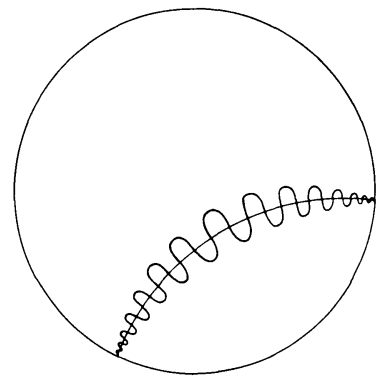

C)

FIG. 6

The two simplest closed orbitlike free elastica in the Poincare disc: (a) $\sigma_{2,3}$, (b) $\sigma_{3,5}$.

In the orbitlike case one again obtains a countably infinite family of closed solutions $\sigma_{m, n}$. Here $m>1$ is an integer and $n$ is an integer satisfying $\frac{1}{2}<m / n<\sqrt{2} / 2$. This condition follows from another elliptic integral in the appendix, which shows that as $\alpha$ increases from its lower limit $-2 G$ to its upper limit $-4 G$, the rotation, $\Delta \theta$, of $\sigma$ decreases monotonically from $\sqrt{2} \pi$ to $\pi$.

In contrast with the wavelike case, a closed free orbitlike elastica $\sigma$ is always null-homotopic. For suppose $\tilde{\sigma}$ is a lift of $\sigma$ to the universal cover $\tilde{M}$ of $M$. Then $\tilde{\sigma}$ lies inside a compact subset of $\tilde{M}$-namely that bounded by the invariant circle $\Sigma^{+}$(see Figure 2). So $\sigma$ cannot represent an element of infinite order in $\pi_{1}(M)$; but $\pi_{1}(M)$ is torsion free; hence $\sigma$ is null-homotopic. Thus, we might as well always think of the $\sigma_{m, n}$ as lying in $H^{2}$, as illustrated in Figure 7.

Finally, since the asymptotically geodesic elastica $\eta$ is obviously not closed, it remains only to discuss the circular free elastica $\varepsilon$. We refer to $\varepsilon$ as "the equator of the hyperbolic plane" because in some sense it plays the same role in $H^{2}$ as the equator does on the sphere. For just as a circle on $S^{2}$ of arbitrary initial radius will expand to become an equator under the flow of “- $-\nabla \mathfrak{F}$ ", so will a circle in $H^{2}$ expand or contract to achieve the preferred radius of $\varepsilon$ (in this connection, we note that the equator on the sphere is a geodesic circle of radius $\left(\sin ^{-1} 1\right) / \sqrt{G}$, while $\varepsilon$ is a geodesic circle of radius $\left.\left(\sinh ^{-1} 1\right) / \sqrt{-G}\right)$.

More generally, one interpretation of the following result is that any "initial" curve $\gamma$ which is regularly homotopic to $\varepsilon$ ought to flow to $\varepsilon$ :

Theorem 4.1. Let $M^{2}$ be a manifold with constant curvature $G<0$. Then any null-homotopic regular closed curve $\gamma$ in $M$ satisfies $\int_{\gamma} k^{2} d s \geqslant 4 \pi \sqrt{-G}$, and equality holds if and only if $\gamma$ is congruent to $\varepsilon$. 


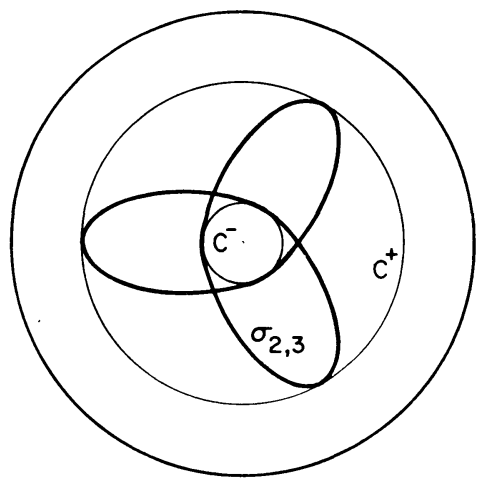

a)

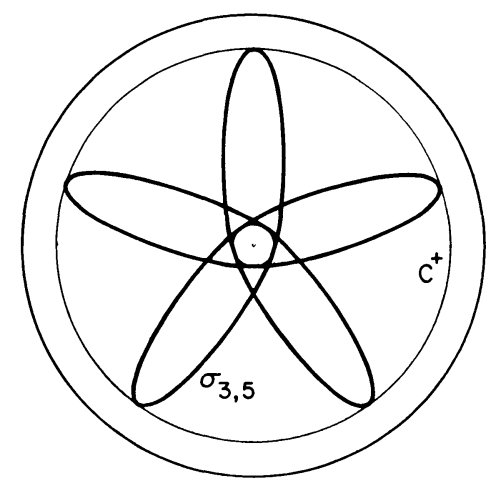

b)

FIG. 7

Conjecture. Let $M^{n}$ be a Riemannian manifold with sectional curvature bounded above by $G<0$. If there exists a regular closed curve $\gamma$ in $M^{n}$ satisfying $\int_{\gamma} k^{2} d s<4 \pi \sqrt{-G}$, then $M^{n}$ is not simply connected.

We defer the proof of Theorem 4.1 until the end of this section so that we may first point out some interesting facts surrounding this result. To begin with, it is natural to suppose that $\varepsilon^{m}$-the $m$-fold cover of $\varepsilon$-minimizes $\mathfrak{F}$ in its regular homotopy class. Such a view is shattered by

Theorem 4.2. The $m$-covered equator $\varepsilon^{m}$ in $H$ is stable for $|m|=1,2$ and unstable for $|m| \geqslant 3$.

Proof. For $\gamma=\varepsilon^{m}, k=\sqrt{-2 G}$, so the second variation formula gives $\delta^{2} \widetilde{F}=2 \int_{\gamma}(\ddot{\phi}-2 G \phi)(\ddot{\phi}-G \phi) d s$. Since $\varepsilon^{m}$ has length $2 \pi m / \sqrt{-G}, \phi$ must have Fourier series of the form

$$
a_{0} / 2+\sum_{1}^{\infty} a_{n} \cos (n \sqrt{-G} s / m)+b_{n} \sin (n \sqrt{-G} s / m) .
$$

Computing $\ddot{\phi}$ and substituting $\phi, \ddot{\phi}$ into the above formula yields

$$
\delta^{2} \mathfrak{F}=2 \pi m(-G)^{3 / 2}\left[a_{0}^{2}+\sum_{1}^{\infty}\left(n^{2} / m^{2}-1\right)\left(n^{2} / m^{2}-2\right)\left(a_{n}^{2}+b_{n}^{2}\right)\right] .
$$

Now consider first the case $|m|=1,2$. Then one easily sees that $\delta^{2} \mathfrak{F} \geqslant 0$, with equality occurring precisely when $a_{n}=b_{n}=0, n \neq m$, and $\phi=$ $a_{m} \cos \sqrt{-G} s+b_{m} \sin \sqrt{-G} s$. Such $\phi$ make up the two-dimensional space of normal Killing fields along $\gamma$. Thus we have stability for $\varepsilon^{2}$ as well as for $\varepsilon$ (the latter being already known). 
Now suppose $|m| \geqslant 3$. In this case, we can make $\delta^{2} \mathfrak{F}$ negative by setting $\phi=\cos (\sqrt{-G}(m+1) s / m)$, so $\varepsilon^{m}$ is unstable. q.e.d.

This leads one to ask if, instead $\mathfrak{F}$ is minimized (among curves regularly homotopic to $\varepsilon^{m}$ ) by an orbitlike elastica (the only other kind of closed free elastica in the same class). However, a computation involving elliptic integrals (see the appendix) proves $\mathfrak{F}\left(\sigma_{m, n}\right)>\mathfrak{F}\left(\varepsilon^{m}\right)$. Thus we have the

Corollary. F restricted to the regular homotopy class of $\varepsilon^{m},|m| \geqslant 3$, does not achieve its infimum. Moreover, if $\gamma_{i}$ is a minimizing sequence for $\mathfrak{F}$ so restricted, then the lengths of $\gamma_{i}$ must approach infinity.

(The last sentence depends on a compactness result which is stated in the proof of Theorem 4.1.)

Figure 8 shows, for the case $m=3$, how $\mathfrak{L}\left(\gamma_{i}\right)$ can be arbitrarily large while $\mathfrak{F}\left(\gamma_{i}\right)$ remains bounded. The curve consists of four geodesic segments of length $i$, capped off by four arcs of equators $\varepsilon$. The proof of Theorem 4.2 suggests why this four-fold symmetry is appropriate here.

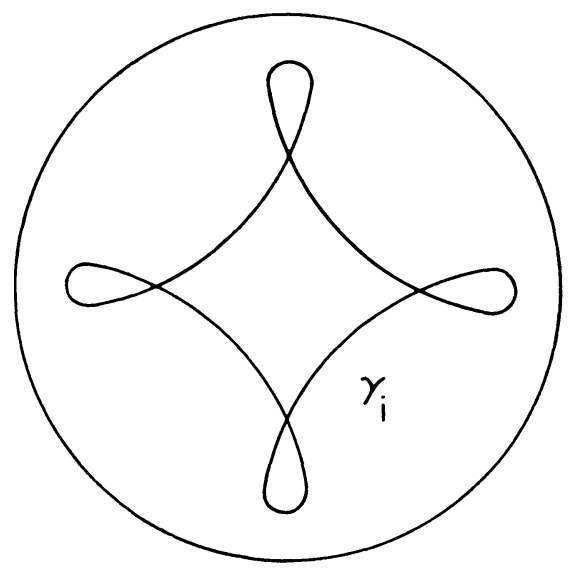

FIG. 8

Another phenomenon to be noted in connection with Theorem 4.1 is that if one considers the problem of minimizing $\mathfrak{F}$ subject to the constraint of fixed length $l$, one obtains a circle $C_{l}$ for some values of $l$, yet clearly not for large values of $l$. In fact, the proof of Theorem 4.1 implies the following "restricted isoperimetric inequality":

Corollary. There exists a constant $L>\mathfrak{L}(\varepsilon)=2 \pi / \sqrt{-G}$ such that any regular simple closed curve $\gamma$ in $H$ of length $l<L$ satisfies $\int_{\gamma} k^{2} d s \geqslant 4 \pi^{2} / l-G l$. 
Proof of Theorem 4.1. Let $\gamma_{0}$ be a regular closed curve in $H$, and let $\Omega$ be the set of regular closed curves of length at most $\mathfrak{L}\left(\gamma_{0}\right)$. Then to verify the inequality for $\gamma_{0}$, it suffices to show:

(1) there exists a closed elastica $\gamma$ (not necessarily free) minimizing $\mathfrak{F}$ over $\Omega$,

(2) $\gamma$ must be simple, and

(3) all simple closed elasticae satisfy the inequality, with equality only for $\gamma=\varepsilon$.

We discuss these in reverse order, the crucial one being (3). Observe first that of the five types of elasticae, it suffices to consider circles and orbitlike elasticae, since the others are either not closed or not simple (a closed wavelike elastica has rotation index 0 ).

If $\gamma$ is a circle of radius $r$, then

$$
\begin{aligned}
\mathfrak{L}(\gamma) & =\frac{2 \pi}{\sqrt{-G}} \sinh \sqrt{-G} r, \quad \mathscr{A}(\gamma)=\frac{2 \pi}{-G}(\cosh \sqrt{-G} r-1), \\
k & =\sqrt{-G} \operatorname{coth} \sqrt{-G} r, \quad \mathfrak{F}(\gamma)=2 \pi \sqrt{-G} \frac{\cosh ^{2} \sqrt{-G} r}{\sinh \sqrt{-G} r} .
\end{aligned}
$$

Thus, $\mathfrak{F}(\gamma)$ is smallest when $\sinh \sqrt{-G} r=1$, i.e., $\gamma=\varepsilon$, and $\mathfrak{F}(\gamma)=4 \pi \sqrt{-G}$.

Now suppose $\gamma$ is orbitlike, hence $k^{2}=\alpha \operatorname{dn}^{2}(r s, p)$ and $r=\sqrt{\alpha} / 2, \alpha=$ $(2 \lambda-4 G) /\left(2-p^{2}\right)$. If $\gamma$ closes us in $n$ periods, one can easily obtain the estimate (see first paragraph of the appendix)

$$
\mathfrak{F}(\gamma)=\frac{2 n}{r} \int_{0}^{K} \alpha \mathrm{dn}^{2}(u) d u>4 n \sqrt{2 \lambda-4 G} .
$$

Thus, we need to estimate $\lambda$ and $n$ from below.

If $A$ is the area bounded by $\gamma$, the Gauss-Bonnet formula gives

$$
0<-G A=\int_{\gamma} k d s-2 \pi=\frac{2 n}{r} \int_{0}^{K} \sqrt{\alpha} \operatorname{dn}(u, p) d u-2 \pi=2 \pi(n-1),
$$

so for one thing $n \geqslant 2$. Note that this implies $J_{\gamma}$ is a rotation field; for if $J_{\gamma}$ were a translation or horocycle field, $\gamma$ would have to close up in one period or not at all. Thus, by Proposition 2.2, there exists a circle $C^{-}$inscribed in $\gamma$ and having curvature $k_{C}=-2 G \sqrt{\alpha_{2}} /\left(\alpha_{2}-\lambda\right)$. Since area $\left(C^{-}\right)<A$, a comparison of Gauss-Bonnet Formulas for $\gamma$ and $C^{-}$leads to $k_{C}^{2} \leqslant n^{2} k_{C}^{2}+n^{2} G$, and hence

$$
\frac{-n^{2} G}{n^{2}-1} \leqslant k_{C}^{2}=\frac{4 G^{2} \alpha_{2}}{\left(\alpha_{2}-\lambda\right)^{2}}=\frac{4 G^{2}}{\left(\sqrt{\alpha_{2}}-\lambda / \sqrt{\alpha_{2}}\right)^{2}} \text {. }
$$

It follows that

$$
\lambda \geqslant-\frac{1}{4}\left(\sqrt{\alpha_{2}}-\frac{\lambda}{\sqrt{\alpha_{2}}}\right)^{2} \geqslant G\left(\frac{n^{2}-1}{n^{2}}\right) .
$$


Substitution into the original estimate for $\mathfrak{F}(\gamma)$ now gives $\mathfrak{F}(\gamma) \geqslant$ $4 \sqrt{-G} \sqrt{2 n^{2}+2} \geqslant 4 \sqrt{-G} \sqrt{10}>4 \pi \sqrt{-G}$.

For the proof of (2), we restrict our attention to wavelike $\gamma$, since the orbitlike case is much the same (a more careful version of (3) would also take care of nonsimple orbit-like $\gamma$ ). For $\gamma$ to close up, its wavelength must be 0 , so $\gamma(0)=\gamma(2 K / r)$, and $\gamma$ closes up smoothly in one period to form a figure eight.

Let $\Gamma^{\prime}$ be the geodesic through $p=\gamma(0)$ crossing the axial geodesic $\Gamma$ perpendicularly. By symmetry of $\gamma(k(s)$ is even about $K / r), \Gamma^{\prime}$ divides $\gamma$ into two congruent halves (see Figure 9). Let $q$ be one of the points on $\gamma$ of maximum distance from $\Gamma^{\prime}$, and $\bar{q}$ its reflection through $\Gamma^{\prime}$. Then the geodesic $\Gamma^{\prime \prime}$ passing through $q$ and $\bar{q}$ must cross $\gamma$ perpendicularly at both $q$ and $\bar{q}$. The reflection of the upper part of $\gamma$ through $\Gamma^{\prime \prime}$ yields a $C^{1}$-closed curve $\bar{\gamma}$ whose length and total squared curvature are clearly less than that of $\gamma$.

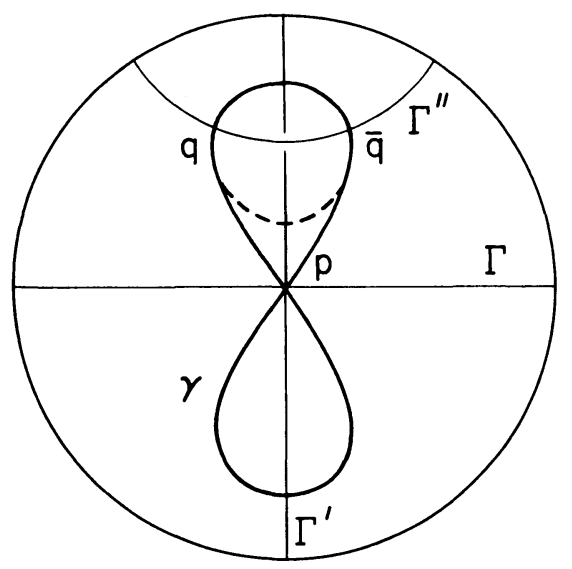

FIG. 9

The proof of (1) is an easy application of the classical direct method in the calculus of variations. We omit the details of this argument, but note that the constraint on arclength is essential for norm-boundedness of $\mathfrak{F}$-bounded sequences $\left\{\gamma_{i}\right\}$ (recall Figure 8).

\section{Appendix}

In order to obtain quantitative information about the total squared curvature $\mathfrak{F}$ of a closed elastica, the wavelength of a wavelike elastica, and the rotation $\Delta \theta$ of an orbitlike elastica, we appeal to standard formulas expressing 
these elliptic integrals in terms of the known functions $K, E, Z, \Lambda_{0}$ - the complete elliptic integrals of the first and second kinds, the Jacobi zeta function, and the Heuman lambda function, respectively (see [3]). Furthermore, we repeatedly make use of the fact that the derivatives of $K, E, Z, \Lambda_{0}$ with respect to the modulus $p$ can be conveniently expressed in terms of these same functions. For instance, in the proof of Theorem 4.1, we needed the following estimate: the facts

$$
E(0)=\frac{\pi}{2}, \quad \frac{d E}{d p}=\frac{E-K}{p} \quad \text { and } \quad \frac{d K}{d p}=\frac{E-\left(1-p^{2}\right) K}{p\left(1-p^{2}\right)}
$$

easily imply $E(p)>\sqrt{2-p^{2}}$, hence the total squared curvature of an orbitlike elastica $\sigma$ of $n$ periods satisfies

$$
\begin{aligned}
\mathfrak{F}(\sigma) & =4 n \sqrt{\frac{2 \lambda-4 G}{2-p^{2}}} \int_{0}^{K} \operatorname{dn}^{2}(u) d u \\
& =4 n \sqrt{\frac{2 \lambda-4 G}{2-p^{2}}} E(p)>4 n \sqrt{2 \lambda-4 G} .
\end{aligned}
$$

We now take up the computation of wavelength.

Proposition 5.1. Let $\gamma$ be a wavelike free elastica. If $G=0$, then

$$
\Lambda=\frac{4 \sqrt{2}}{\sqrt{\alpha}} \int_{0}^{K(\sqrt{2} / 2)} \operatorname{cn}^{2}\left(u, \frac{\sqrt{2}}{2}\right) d u
$$

and if $G \neq 0$,

$$
\Lambda=\frac{4 \sqrt{1-p^{2}}}{p} \sqrt{\frac{1-2 p^{2}}{G}} \int_{0}^{K(p)} \frac{\operatorname{cn}^{2} u d u}{1-\left(\left(2 p^{2}-1\right) / p^{2}\right) \operatorname{sn}^{2} u},
$$

where $p^{2}=\alpha /(2 \alpha+4 G)$.

Proof. Referring to the discussion in $\$ 2$ and using equations (2.1), (2.5), (2.6), and the fact that $k=\alpha \operatorname{cn}(r s, p)$ has period $4 K(p) / r$, we obtain

$$
\begin{aligned}
\Lambda & =\delta x=\int_{0}^{K / r} \frac{4}{\sqrt{\alpha \alpha_{1}}} \frac{T \cdot J}{|J|^{2}} d s=\frac{8 \sqrt{\alpha \alpha_{1}}}{\sqrt{\alpha+\alpha_{1}}} \int_{0}^{K} \frac{\operatorname{cn}^{2}(u, p) d u}{\alpha_{1}-4 G \operatorname{cn}^{2}(u, p)} \\
& =8 \sqrt{\alpha_{1}} p \int_{0}^{K} \frac{\operatorname{cn}^{2}(u, p) d u}{\alpha_{1}-4 G+4 G \operatorname{sn}^{2}(u, p)}
\end{aligned}
$$


For $G=0$, the result is now obvious, and for $G \neq 0$, the stated formula follows easily from the substitutions

$$
\alpha=\frac{4 G p^{2}}{1-2 p^{2}}, \quad \alpha_{1}=\frac{4 G\left(1-p^{2}\right)}{1-2 p^{2}} .
$$

Proposition 5.2. (i) If $G>0$,

$$
\Lambda=\frac{2}{\sqrt{G}}\left[\pi \Lambda_{0}(\phi, p)-\frac{2 p \sqrt{1-2 p^{2}}}{\sqrt{1-p^{2}}} K(p)\right]
$$

where $\phi=\sin ^{-1}\left(\sqrt{1-2 p^{2}} /\left(1-p^{2}\right)\right)$. As $\alpha$ increases from 0 to $\infty$ (so $p$ increases from 0 to $\sqrt{2} / 2$ ), $\Lambda$ decreases monotonically from $2 \pi / \sqrt{G}$ to 0 .

(ii) If $G=0$,

$$
\Lambda=\frac{8 \sqrt{2}}{\sqrt{\alpha}}\left(E\left(\frac{\sqrt{2}}{2}\right)-\frac{1}{2} K\left(\frac{\sqrt{2}}{2}\right)\right),
$$

so $\Lambda$ decreases monotonically from $\infty$ to 0 as $\alpha$ increases from 0 to $\infty$.

(iii) If $G<0$,

$$
\Lambda=\frac{4 K(p)}{\sqrt{-G}}\left[\frac{\sqrt{1-p^{2}} \sqrt{2 p^{2}-1}}{p}-Z(\beta, p)\right],
$$

where $\beta=\sin ^{-1}\left(\sqrt{2 p-1} / p^{2}\right)$. In this case, $\Lambda$ is not monotonic; as $\alpha$ increases from $-4 G$ to $\infty$ (so $p$ decreases from 1 to $\sqrt{2} / 2$ ), $\Lambda$ increases from 0 to its maximum value $b=\Lambda\left(p_{0}\right)$, where $p_{0}$ is the unique solution to $0=2 E(p)-$ $K(p)$, and then $\Lambda$ decreases back down to 0 . The maximum wavelength $b$ is $\approx 2 / \sqrt{-G}$.

Proof. The above formulas for $\Lambda$ follow easily from the previous proposition, and formulas 312.02, 410.03, 414.03 of [3]. The behavior of $\Lambda$ as $\alpha$ approaches its upper and lower limits can then be read off from the limiting behavior of $K, E, Z, \Lambda_{0}$. In the case $G=0$, one could argue instead

The monotonicity of $\Lambda$ for $G>0$ can be established by a straightforward but tedious computation which yields

$$
\frac{d \Lambda}{d p}=\frac{4 / \sqrt{G}}{\sqrt{1-2 p^{2}} \sqrt{1-p^{2}}}[K(p)-2 E(p)]
$$

since $p^{2}<\frac{1}{2}$, this quantity is negative. 
A similar computation for the case $G<0$ gives

$$
\frac{d \Lambda}{d p}=\frac{4 / \sqrt{-G}}{\sqrt{2 p^{2}-1} \sqrt{1-p^{2}}}[2 E(p)-K(p)]
$$

For $\frac{1}{2}<p^{2}<1$, the equation $0=2 E(p)-K(p)$ has a unique solution, hence $\Lambda$ behaves as indicated above. q.e.d.

Using Propositions 2.2 and 2.3 and computations similar to those above, we obtain the corresponding information about the rotation of an orbitlike elastica:

Proposition 5.3. The rotation of an orbitlike free elastica is

$$
\begin{aligned}
\Delta \theta_{\sigma} & =2\left(1-p^{2}\right) \sqrt{2-p^{2}} \int_{0}^{K(p)} \frac{\operatorname{dn}^{2} u}{1-p^{2}\left(2-p^{2}\right) \operatorname{sn}^{2} u} d u \\
& =2 \sqrt{1-p^{2}} \sqrt{2-p^{2}} K(p)+\pi\left[1-\Lambda_{0}\left(\sin ^{-1} \sqrt{1-p^{2}}, p\right)\right] .
\end{aligned}
$$

As $\alpha$ increases from its lower limit $-2 G$ to its upper limit $-4 G, \Delta \theta$ decreases monotonically from $\sqrt{2} \pi$ to $\pi$.

Finally, a comparison of the formulas given here for the total squared curvature $\mathfrak{F}(\sigma)$ and rotation $\Delta \theta_{\sigma}$ of an orbitlike elastica yields the inequality $\mathfrak{F}\left(\sigma_{m, n}\right)>\mathfrak{F}\left(\varepsilon^{m}\right)$ needed for obtaining the corollary to Theorem 4.2.

\section{References}

[1] G. D. Birkhoff, Dynamical systems with two degrees of freedom, Trans. Amer. Math. Soc. 18 (1917) 199-300.

[2] R. Bryant \& P. Griffiths, Reduction for constrained variational problems and $\int k^{2} / 2 d s$, Amer. J. Math., to appear.

[3] P. F. Byrd \& M. D. Friedman, Handbook of elliptic integrals for engineers and physicists, Springer, Berlin, 1954.

[4] H. T. Davis, Introduction to nonlinear differential and integral equations, Dover, New York, 1962.

[5] P. Griffiths, Exterior differential systems and the calculus of variations, Birkhäuser, Boston, 1982.

[6] J. Langer \& D. A. Singer, Curves in the hyperbolic plane and mean curvature of tori in 3-space, Bull. London Math. Soc., 16 (1984), to appear.

[7] _ Knotted elastic curves in $R^{3}$, J. London Math. Soc., to appear.

[8] Curve straightening and a minimax argument for closed elastic curves, Topology, to appear.

[9] R. S. Palais, Critical point theory and the minimax principle, Global Analysis, Proc. Sympos. Pure Math., Vol. 15, Amer. Math. Soc., Providence, RI, 1970, 185-212. 
[10] S. Smale, Regular curves on Riemannian manifolds, Trans. Amer. Math. Soc. 87 (1958) 492-512.

[11] C. Truesdell, The influence of elasticity on analysis: the classical heritage, Bull. Amer. Math. Soc. 9 (1983) 293-310.

MaX - Planck - Institut FÚr Mathematik CASE WeStern Reserve UNIVERSITY 